\title{
Canvas Presentations in the Wild
}

Leonhard Lichtschlag

RWTH Aachen University

lichtschlag@cs.rwth-aachen.de

\section{Thomas Hess}

RWTH Aachen University

hess@cs.rwth-aachen.de

\section{Thorsten Karrer}

RWTH Aachen University

karrer@cs.rwth-aachen.de

\section{Jan Borchers}

RWTH Aachen University

borchers@cs.rwth-aachen.de
Copyright is held by the author/owner(s).

CHI'12, May 5-10, 2012, Austin, Texas, USA.

ACM 978-1-4503-1016-1/12/05.

\begin{abstract}
Most presentation software uses the slide deck metaphor to create visual presentation support. Recently, canvas presentation tools such as Fly or Prezi have instead begun to use a zoomable free-form canvas to arrange information. The effect of this change in format on the authoring process of presentations has been investigated previously in a formal lab study. We have now examined the evolving patterns of usage in publicly available canvas presentations and found that the benefits of this format that have been demonstrated in the lab setting also can be observed in real life presentations. This confirms the potential of canvas based tools to help authors improve the quality of their presentation visuals.
\end{abstract}

\section{Keywords}

Canvas presentations; ZUIs; authoring; study

\section{ACM Classification Keywords}

H.5.2 [Information Interfaces and Presentation (e.g. HCI)]: User Interfaces-Graphical User Interfaces (GUI)

\section{Introduction}

Slide-based visual presentation support, such as Microsoft's PowerPoint or Apple's Keynote, is prevalent when looking at talks in research, industry, education, government, and many other areas. But, this format has been criticized repeatedly for the limitations it imposes on authors and presenters $[6,8]$. 
(1)

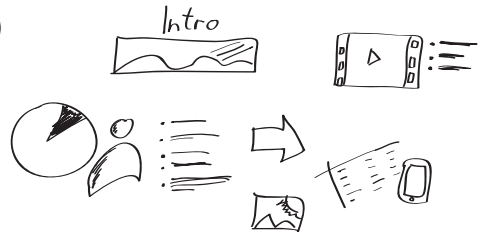

(2)

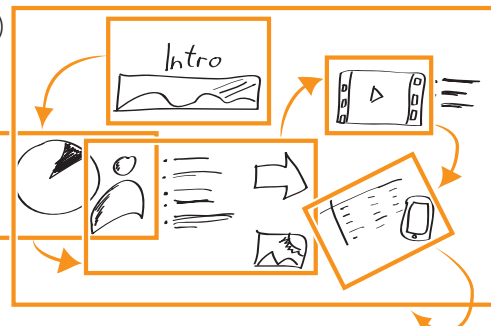

(3)

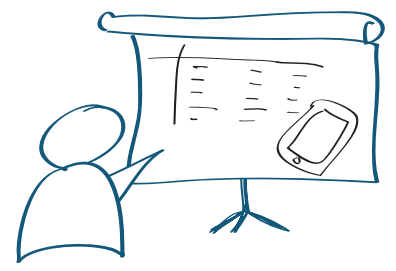

Figure 1. A workflow of authoring canvas presentations. (1) The author arranges content in a spatial way. (2) She adds a series viewports over the canvas that form the presentation sequence. Varying zoom depths show different amounts of content at once. (3) A viewport during presentation delivery.
Among the attempts to mitigate the problems of slideware are canvas presentations, e.g., Prezi ${ }^{1}$, which dismiss the slide metaphor in parts or entirely. Instead, presentation authors place either the slides [2] or their individual elements $[4,5]$ on an infinite canvas. They then define viewports and transition paths across the canvas to create the presentation sequence. In this paper, we present the results of a case study [3], in which we investigated publicly available canvas presentations. Our goal was to analyze how authors use these new tools and handle their capabilities in creating presentation documents for their own demands.

\section{Authoring a Canvas Presentation}

While early zoomable user interfaces [1] were also used in talks occasionally, several tools have been designed specifically with presentation support in mind $[2,4,5]$. CounterPoint [2] broke new ground by allowing the author to position PowerPoint slides inside a zoomable user interface. These slides are placed at varying distances from a virtual camera, thus creating a spatial layout that in itself reflects the macrostructure of the talk. Overviews can be created easily by zooming out and presenting the slide arrangement as a whole to the audience. This model was also adopted by pptPlex ${ }^{2}$

Fly [5] and Prezi completely abandoned the slide metaphor, letting authors put content elements (text, figures, etc.) directly on a canvas (fig. 1). Thus, the contents are no longer bound to the slide frame, and do not need to be split into fixed-size chunks. A presentation is defined as a path across the planar canvas with path stops that show views of the canvas. In Prezi, viewports are associated with either a content or a frame element and can be rotated.

\footnotetext{
1 www.prezi.com $\quad 2$ www.officelabs.com/projects/pptPlex
}

Two studies [5] examined the process of authoring canvas presentations with Fly compared to using the traditional slide deck format. They showed that the resulting Fly documents tended to be more diversified and better represented the structure of connected topics. In contrast to slides, where authors have to map their content to a linear sequence of equally-sized chunks, the canvas method makes it easier to present complex topics that have more than one logical dimension or are highly interconnected $[2,5]$. Authors also embraced the possibilities of the non-linear layout of information and expressed a clear preference for canvas layouts. We are validating these results by investigating how authors use these new canvas tools in everyday practice.

\section{Study Method}

We examined a pool of Prezi documents to see how authors use a canvas-based presentation format for real world tasks. With Prezi, documents that are created using a free account are publicly accessible on the 'Explore' section of the Prezi website ${ }^{1}$, either read-only or even available for reuse by others. For this evaluation, we considered the most popular 73 of the 308 presentations listed on July 1, 2010. While this may not be representative for all canvas presentations, it helped us to concentrate on documents that were considered well-authored. We excluded documents that were either clearly not created as live presentation support, not finished, or served as instructions for Prezi, so that 50 presentations remained. These presentations were examined with regards to use of layout strategies, overviews, zooming, and rotations.

\section{Study Findings}

The first thing to note is that every document studied had a unique canvas layout; there were no recurring 


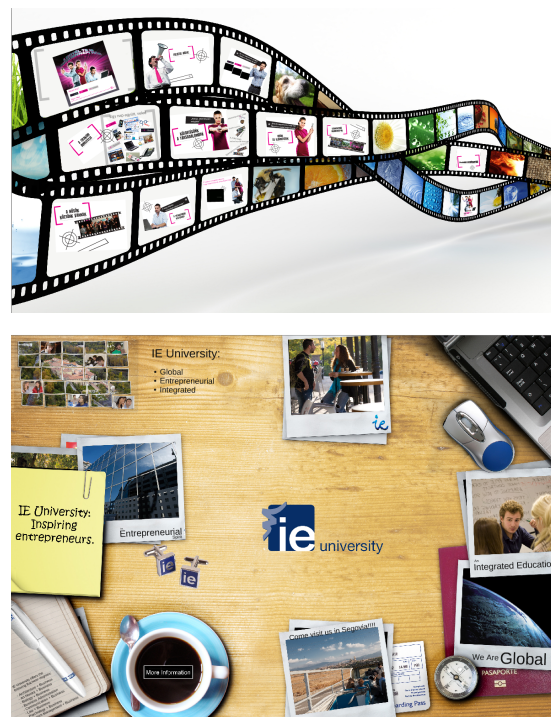

Figure 2. Prezi canvases with decorative layouts. Top: viewports are embedded into a film strip. Bottom: a large photo of a desktop is used in the background. designs as it is common with slide presentations. Nearly all authors utilized scaling and zooming to achieve varying viewport resolutions, like we expected from the Fly user study [5]. All presentation paths zoomed in on single or few elements to focus on the currently relevant information. Other common practices for focusing were to zoom in on details of large graphics, such as diagrams and screenshots, or on single words and phrases of larger texts for emphasis.

\section{Layout Strategies}

A recurring strategy are decorative layouts, which use a large background graphic with content elements placed at lower scales into the gaps of the graphic (fig. 2); the content is primarily arranged around the graphic shape. A distinction can be made between this layout and structural layouts, where the arrangement of the content elements reflects the macrostructure of the topic. Out of the examined documents, 36 had structural layouts and 14 had decorative layouts

(fig. 3). For the documents with structural layouts, we identified three subtypes: topic areas, development of an idea, and slide deck layout.

The majority of the documents (29) organized content into topic areas (fig. 4), and the presentation paths explored these areas sequentially. Starting from an overview to preview the upcoming content, such a path drilled into one topic and then, after covering it completely, zoomed back out-either showing a repeated overview of the past topic for recapitulation or directly moving on to an overview of the next topic-and then drilled into the next topic. This kind of structure was often built recursively with topics that contained subtopics, which were traversed in the same way.

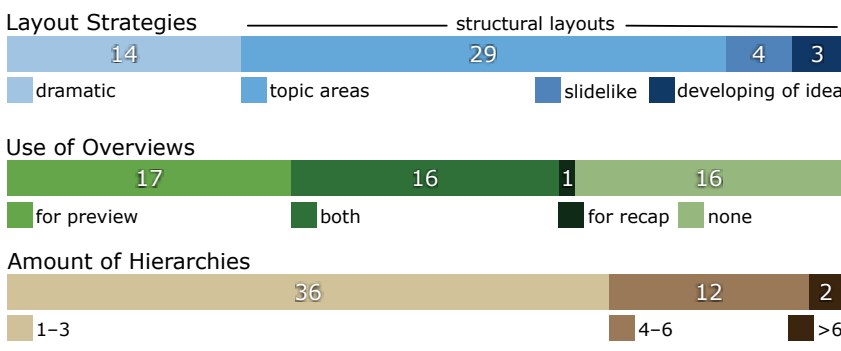

Figure 3. Distribution for layout strategies, amount of zoom levels, and use of overviews for the examined presentations.

Three documents had structures that incrementally developed an idea (fig. 5). Their presentation paths started by showing content on a detail scale. Then, they continuously zoomed out, incrementally revealing more content, ending with a view of the whole canvas. Accordingly, the content was scaled larger the later it occurred in the presentation sequence. Overviews were mainly used to recapitulate.

Four documents had structures similar to slide decks. All the content elements shared a small range of zoom levels and the paths traversed them sequentially while constantly remaining on the detail zoom levels with none or little overviews. Purpose of these presentations was to tell a story as opposed to inform about a topic.

\section{Overviews}

The majority of presentations (33) utilized overviews in their paths: 17 used overviews to preview and recapitulate content; 16 used overviews only to preview; one used overviews only to recapitulate (fig. 3 ).

\section{Zooming}

Because of the unlimited zooming capabilities of the Prezi canvas, there was no restriction on how deep the 

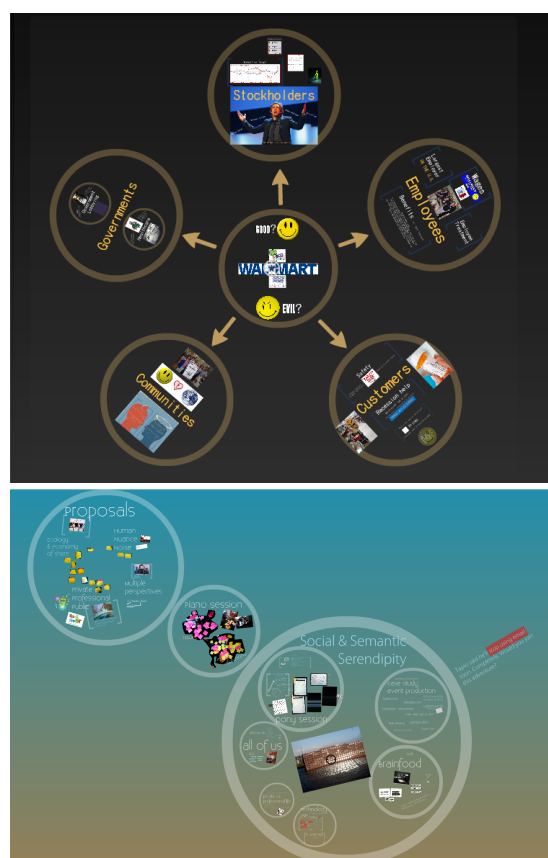

Figure 4. Two Prezi canvases with topic area structures

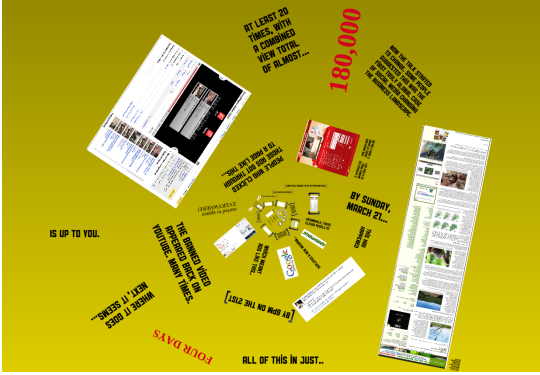

Figure 5. This presentation incrementally develops of an idea by zooming out content hierarchies could be nested or how small content elements could be created. However, 36 of the examined documents did not create hierarchies deeper than three levels (e.g., all content, topics, and subtopics). Among the others, 12 had hierarchies of four to six hierarchy levels and only two of the presentations that developed an idea had more than six levels as a result of their canvas structure (fig. 3). Another recurring pattern is using unlimited zooming to hide content, such as a footnote to a text, by scaling it down smaller than a recognizable scale. In the presentation path a dramaturgic zoom-in-movement reveals the hidden content.

\section{Rotation}

Three of the presentations used rotation in meaningful ways, e.g., when the content had circular arrangements. However, 29 presentations used rotation primarily to achieve decorative canvas layouts or to provoke impressive viewport transitions-elements were often rotated by $90^{\circ}$ or more or in opposed directions. In decorative layouts, content elements were often rotated to make them fit into the intended shape (fig. 2).

\section{Discussion}

Compared to Lichtschlag et al.'s lab study [5], we found similar results: We also noted a use of diverse layout strategies grounded in the macrostructure of the content. Lichtschlag et al. found that the canvas format facilitates employing an expressive layout for the purpose of overviews; we could confirm this observation with our selected body of presentations. The frequent use of hierarchies (fig. 3) indicates that a canvas presentation tool should allow the nesting of content-either via the use of zooming or through explicit layers. Rotation, which was not investigated in [5], was used in the majority of presentations, but mainly for decorative reasons. This may come at a cost, since spatial knowledge acquired from maps is not robust against orientation manipulations [7]. Overall, this study validates the results from the lab setting [5] and shows that the theoretical benefits of canvas presentations do, in fact, influence everyday authoring practices.

\section{Acknowledgements}

This work was funded by the German B-IT Foundation and by the German Government through its UMIC Excellence Cluster for Ultra-High Speed Mobile Information and Communication at RWTH Aachen University.

\section{References}

[1] B. B. Bederson and J. D. Hollan. Pad++: a zooming graphical interface for exploring alternate interface physics. In Proc. UIST 1994, 17-26.

[2] L. Good and B. Bederson. Zoomable user interfaces as a medium for slide show presentations. Information Visualization, 1(1):35-49, 2002.

[3] T. Hess. Fly: Expressive and Conveying Planar Presentations, University of Trento and RWTH Aachen University, Master's Thesis, 2011.

[4] L. Laufer, P. Halacsy, and A. Somlai-Fischer. Prezi Meeting: Collaboration in a Zoomable Canvas Based Environment. In CHI EA 2011, 749-752

[5] L. Lichtschlag, T. Karrer, and J. Borchers. Fly: a Tool to Author Planar Presentations. In Proc. CHI 2009.

[6] I. Parker. Absolute PowerPoint: Can a software package edit our thoughts? The New Yorker, 77(13):76-87, 2001.

[7] D. Schacter and L. Nadel. Varieties of spatial memory: A problem for cognitive neuroscience. Perspectives on cognitive neuroscience, 165-185, 1991.

[8] E. Tufte. The Cognitive Style of PowerPoint. Graphics Press, Cheshire, Connecticut, USA, 2003. 